\title{
Interactive comment on "Required
}

\section{sampling-density of ground-based soil moisture} and brightness temperature observations for calibration/validation of L-band satellite \section{observations based on a virtual reality" by S. Lv et} al.

\section{Anonymous Referee \#2}

Received and published: 30 July 2019

The authors use a virtual reality to estimate minimum requirements for in situ station density for satellite soil moisture and brightness temperature cal/val. The study is well executed, has interesting, impactful findings, and is generally well presented. My lone concern is the lack of consideration of previous, related works and insufficient context for the conclusions presented here. Overall, I recommend the authors revisit the extensive literature devoted to understanding in situ sampling requirements for satellite soil moisture cal/val and provide a better, in-depth synthesis of this literature both in 
the introduction and conclusion/discussion sections.

Specific comments: The introduction is lacking sufficient review of past literature, despite a growing number of studies focused on in situ sampling requirements for satellite soil moisture cal/val. Recent examples include Molero et al 2017, Bhuiyan et al. 2018, and Chen et al. 2019, among others. The statement on lines $75-77$ is technically true, but that does not preclude the authors from developing an in-depth synthesis of previous, related studies. The same can be said for the Conclusion and discussion section, which consists of much more conclusion than discussion. Please expand this section to include a discussion of your findings in the context of previous studies examining sampling density and satellite soil moisture or brightness temperature cal/val, and how your findings build off of and/or improve on these previous studies. Also, please include some discussion of how your findings - in virtual reality - could apply in or differ from reality. For example, most existing in situ networks do not sample soil moisture systematically within a pixel-area, but are often clustered within certain parts of the pixel. How could this affect determination of minimum sampling distances? Given your findings, how many SMAP core validation sites, for example, meet the recommended requirements for sampling distance?

Technical corrections: Line 18: by "better" do you mean "finer"? Lines 37-38: This is vague, yet overreaching statement. Effective in situ soil moisture observation at continental scale is possible depending on the application. Please refine this statement. Line 41: add comma separating "satellite" and "SMOS" Line 48: replace "set up" with "establish" Line 58: "better 15 observations" is oddly used here. Is this a direct recommendation from the reference, or are you simply arguing that more stations are better than fewer stations? Either way, I think it can be deleted without consequence. Lines 61-68: Are these figures from some previous study or official recommendation? How did you arrive at these values? Line 62: should be "should be sampled with at least eight stations" Figure 1: could you report the PFT name along with or instead of the PFT number in the third panel, instead of referencing the PFT name in the caption?

Interactive comment
Printer-friendly version

Discussion paper 
Line 229: replace "only" with "at least", and "70 percentile" should be "70th percentile" Line 323: "thus" is used too often in this sentence. Please throw in a "therefore" instead Line 327: replace "as an ideal footprint" with just "ideal" Lines 335-339: this is quite a long sentence, please consider rephrasing Line 360: should be "that represents" and not "which represents" line 361: add "is necessary" between "3 km" and "if we want..."

Molero, B., et al., 2018: Multi-Timescale Analysis of the Spatial Representativeness of In Situ Soil Moisture Data within Satellite Footprints. JGR: Atmospheres, 123(1), 3-21, https://doi.org/10.1002/2017JD027478

Bhuiyan, et al., 2018: Assessing SMAP Soil Moisture Scaling and Retrieval in the Carman (Canada) Study Site. Vadose Zone Journal, 17(1), https://doi.org/10.2136/vzj2018.07.0132

Chen et al., 2019: Uncertainty of Reference Pixel Soil Moisture Averages Sampled at SMAP Core Validation Sites. Journal of Hydrometeorology, https://doi.org/10.1175/JHM-D-19-0049.1

Interactive comment on Hydrol. Earth Syst. Sci. Discuss., https://doi.org/10.5194/hess-2019192, 2019. 